\title{
Ship Tracking Based on Underwater Electric Potential
}

\author{
Baoquan Sun, ${ }^{1,2}$ Bing Yan, ${ }^{1}$ Jiawei Zhang, ${ }^{1}$ and Shouwei Hu $\mathbb{D}^{2}$ \\ ${ }^{1}$ College of Weapon Engineering, Naval University of Engineering, Wuhan, Hubei 430033, China \\ ${ }^{2}$ Science and Technology on Near-Surface Detection Laboratory, Wuxi, Jiangsu 214035, China \\ Correspondence should be addressed to Shouwei Hu; shouwei_hu_w@yeah.net
}

Received 18 May 2018; Accepted 11 October 2018; Published 12 December 2018

Academic Editor: Nuno Simões

Copyright (C) 2018 Baoquan Sun et al. This is an open access article distributed under the Creative Commons Attribution License, which permits unrestricted use, distribution, and reproduction in any medium, provided the original work is properly cited.

\begin{abstract}
Underwater Electric Potential is an important signal characteristic of a ship. The signal contains location information which can be used to track the ship. This research tries to study the possibility of ship tracking using Underwater Electric Potential. Aiming at the problems existing in the traditional Kalman filters under large initial errors, a new nonlinear filter is proposed. State space model of ship tracking is established; the problem existing in the ordinary Kalman filters is analyzed from the perspective of Kullbeck-Leibler Divergence; the new algorithm is proposed based on progressive Bayesian; simulations are designed. Simulation results show: it is feasible to use underwater electric potential to track the ship; the new method can effectively improve filter performance under large initial error and can effectively track the ship with preferable precision and convergence, which has great practical value.
\end{abstract}

\section{Introduction}

Underwater Electric Potential (UEP) resulting from corrosion or anticorrosion current of a ship is an important signal that is not negligible. There is obvious distribution characteristic with UEP [1] which can be used to track the ship. Currently, studies on UEP mainly focus on the model construction $[2,3]$, and some literatures begin to explore the feasibility of using Static Electric Field (SE) to track ship [4]. With the development of vibration absorption, noise reduction, and electric propulsion technology, ship radiation noise level has been greatly reduced. Coupled with sound line bending, sound reverberation, and other natural conditions, passive acoustic tracking is becoming more difficult. However, UEP signal is less affected by the environment. Ship tracking using UEP can be an effective supplement to acoustic tracking. The present research aims to study the possibility of tracking ship using UEP.

The most common method of target tracking is filtering, which has been studied extensively. Filtering method is an on-line recursive method based on the dynamic model and the observation model that can estimate the target state from the noisy observation data in real time. The main difficulty in the filtering method for ship tracking using UEP is that it is not easy to set filter initial value. However, the initial value is a key to affect the filter performance. In the ship tracking problem, the azimuth-distance information is hidden in the UEP signal, resulting in the initial value being difficult to set, and usually with large initial errors.

Extended Kalman filter (EKF) usually provides good estimation performance, and it has been widely used. However, EKF fails in some applications when the observation model is strong nonlinear [5]. There are several other filters designed to deal with nonlinear problems. Unscented Kalman filter (UKF) [6] and cubature Kalman filter (CKF) [7], typical of the sigma-point class, are able to retain higher-order terms of the Taylor series by choosing various sigma points spread around the distribution function and constructing the filter gain from the sigma points. Another important set of filters are the high-order filters. The second-order filters (SOF) [8] are the best known among them. These filters approximate the models by a high-order Taylor approximation. However, the complexity and computational load of these filters make their implementation unfeasible, particularly in the case where the state variable is high dimensional $[9,10]$.

Particle filters [11] approximate the full probability distribution, by using a finite set of samples. These filters use sequential sampling and resampling according to an "importance function" to provide much better performance than Kalman filters. The improved performance is especially 
pronounced when using nonlinear models or non-Gaussian distributions. However, the high computational cost associated with using the large number of samples required makes them unsuitable for underwater tracking applications.

One way to solve the inherent problem of traditional filters is the use of progressive Gaussian filtering (PGF) [12], which gradually includes the measurement information instead of using all the information in one step. According to this idea, a new GA filter is derived through approximating intermediate progressive joint PDFs of state and measurement as Gaussian in reference [13]. Another way is the use of particle flow filters which propose an alternate convenient way of performing Bayesian updates [14] through probabilistic inference. Particle flow filters rely on particles to carry out, which leads to the same problem as particle filters. A progressive Bayesian method without particles is derived under the Gaussian assumption in [15]. Comparing [13] and [15], we can find that, under the Gauss hypothesis, two methods are consistent in form. In fact, both class filters are just different manifestations of progressive Bayesian.

According to the previous analysis, Kalman filters are more suitable for underwater applications than particle filters. Therefore, consider applying the progressive Bayesian idea to the Kalman filters to improve their performance. Ship motion is of low maneuverability, and its dynamic model can be assumed as a uniform linear motion model. Therefore, the essence of applying progressive Bayesian to Kalman filters lies in redesigning measurement update. Kalman filters perform one measurement update when a measurement is received. The idea behind progressive Bayesian is to introduce the measurement information progressively in pseudo time which equals performing the measurement update of Kalman filters multiple times. So the new methods are named as progressive update Kalman filters (PU-KFs).

The remainder of this paper is organized as followed. In Section 2, we present the ship tracking problem with UEP and analyze the problem existing in the ordinary Kalman filters from the perspective of Kullbeck-Leibler Divergence (KLD). In Section 3, we derive the proposed algorithm based on progressive Bayesian. In Section 4, we design simulations to verify related theory. In Section 5, we draw a simple conclusion for the paper.

\section{Problem Statement}

2.1. State Space Model. The general tracking model consists of the dynamic model and observation model

$$
\begin{aligned}
& \boldsymbol{x}_{k}=\boldsymbol{a}\left(\boldsymbol{x}_{k-1}\right)+\boldsymbol{w}_{k-1} \\
& \boldsymbol{y}_{k}=\boldsymbol{h}\left(\boldsymbol{x}_{k}\right)+\boldsymbol{v}_{k}
\end{aligned}
$$

where $\boldsymbol{x}_{k} \in \mathbb{R}^{n}$ is the $n$-dimensional state vector; $\boldsymbol{y}_{k} \in \mathbb{R}^{m}$ is the $m$-dimensional observation vector; $\boldsymbol{a}$ and $\boldsymbol{h}$ are some known functions; $\left\{\boldsymbol{w}_{k-1}\right\}$ and $\left\{\boldsymbol{v}_{k}\right\}$ are white noises and are independent, $\boldsymbol{w}_{k-1} \sim \mathrm{N}\left(\mathbf{0}, \boldsymbol{Q}_{k-1}\right), \boldsymbol{v}_{k} \sim \mathrm{N}\left(\mathbf{0}, \boldsymbol{R}_{k}\right)$.

Ship UEP is mainly modeled by boundary element method [16], finite element method [17] and equivalent source method [18]. Boundary element method and finite element method cannot reverse the source information from electric field information. By comparison, the equivalent source method has an exact mathematical model. Among the various equivalent source models, point current (a point where current flows in or out) model is closest to the actual ship model and has higher precision [19]. Therefore, the ship UEP signal is modeled by point current method.

According to point current method the ship can be described as a uniform linear array (referred to as point current array) with $N(N \geq 2)$ point currents at equal distance. The current density of each point current is $I_{p i}$ and the distance is $l_{d}$. In general, the larger $N$, the higher accuracy of the target's signal fitting, but at the same time it will lead to an increase in computational complexity. To this end, the choice of $N$ is related to the scale of the target and our requirements for computational accuracy. The UEP at a filed point is the sum of the UEP produced by the $N$ point currents at this field point.

$$
U=\sum_{i=1}^{N} I_{p i} K\left(I_{p i}, P_{\mathrm{f}}\right)
$$

where $K\left(I_{p i}, P_{\mathrm{f}}\right)$ is the distance function between the point current and the field point. In the air-seawater-seabed threelayer uniform media (as shown in Figure 1), $K\left(I_{p i}, P_{\mathrm{f}}\right)$ is

$$
\begin{gathered}
K\left(I_{p i}, P_{\mathrm{f}}\right)=\frac{1}{4 \pi \sigma}\left\{\frac{1}{\sqrt{r^{2}+\left(z-z_{i}\right)^{2}}}\right. \\
+\frac{1}{r^{2}+\left(z+z_{i}-h\right)^{2}} \\
\left.+\sum_{m=1}^{\infty}\left[\begin{array}{c}
\frac{1}{\sqrt{r^{2}+\left(z+z_{i}-h+2 m H\right)^{2}}} \\
+\frac{1}{\sqrt{r^{2}+\left(z+z_{i}-h-2 m H\right)^{2}}} \\
+\frac{1}{\sqrt{r^{2}+\left(z-z_{i}+2 m H\right)^{2}}} \\
+\frac{1}{\sqrt{r^{2}+\left(z-z_{i}-2 m H\right)^{2}}}
\end{array}\right]\right\}
\end{gathered}
$$

where $H$ is the depth of seawater; $h$ is the depth of sensor; $r^{2}=\left(x-x_{i}\right)^{2}+\left(y-y_{i}\right)^{2} ; \sigma$ is the seawater conductivity; $\sigma_{1}$ is the seabed conductivity; $k=\left(\sigma-\sigma_{1}\right) /\left(\sigma+\sigma_{1}\right)$ is the seabed reflection coefficient; $m$ is the number of reflections; and the limit in calculation is usually 10 20 [20]. 




FiguRE 1: Air-seawater-seabed three-layer uniform media.

UEP measured at the N0.j electric field sensor can be modeled as

$$
y^{(j)}\left(\boldsymbol{x}_{k}\right)=h^{(j)}\left(\boldsymbol{x}_{k}\right)+v_{k}^{(j)}=U_{k}^{(j)}+v_{k}^{(j)}
$$

where $v_{k}^{(j)}$ is the measured noise.

And then, the observation equation is

$$
\boldsymbol{y}_{k}=\boldsymbol{h}\left(\boldsymbol{x}_{k}\right)+\boldsymbol{v}_{k}=\left[\begin{array}{c}
U_{k}^{(1)} \\
U_{k}^{(2)} \\
\vdots \\
U_{k}^{(J)}
\end{array}\right]+\left[\begin{array}{c}
v_{k}^{(1)} \\
v_{k}^{(2)} \\
\vdots \\
v_{k}^{(J)}
\end{array}\right]
$$

The state vector contains the target position, velocity, and point current array, while the motion of the ship's $Z$ direction is neglected.

$$
\boldsymbol{x}_{k}=\left[\boldsymbol{r}_{k}, \boldsymbol{V}_{k}, I_{\mathrm{p}}^{1: N}\right]^{\mathrm{T}}
$$

where $\boldsymbol{r}_{k}=[x, y, z]^{\mathrm{T}}, \boldsymbol{V}_{k}=\left[V_{x}, V_{y}\right]^{\mathrm{T}}$, and $I_{\mathrm{p}}^{1: N}=$ $\left[I_{\mathrm{p} 1}, I_{\mathrm{p} 2}, \ldots, I_{\mathrm{p} N}\right]^{\mathrm{T}}$.

Ship target has low dynamic characteristics and its motion state can be modeled as Discrete White Noise Acceleration (DWNA) model [21]. The motion equation is

$$
\boldsymbol{x}_{k}^{(r, V)}=\Phi_{m} \boldsymbol{x}_{k-1}^{(r, V)}+\boldsymbol{\Gamma}_{m} \boldsymbol{w}_{k-1}^{(r, V)}
$$

where $\boldsymbol{\Phi}_{m}=\left[\begin{array}{cc}\mathbf{I}_{3 \times 3} & T_{\mathbf{s}} \mathbf{I}_{2 \times 2} \\ \mathbf{0}_{2 \times 3} & \mathbf{I}_{2 \times 2}\end{array}\right]$ is motion state transition matrix, $\boldsymbol{\Gamma}_{m}=\left[\begin{array}{c}\left(T_{s}^{2} / 2\right) \mathbf{I}_{2 \times 2} \\ \mathbf{0}_{1 \times 2} \\ T_{s} \mathbf{I}_{2 \times 2}\end{array}\right]$ is noise gain matrix, and $T_{s}$ is sampling interval.

The DWNA model assumes that the acceleration is Gaussian white noise acceleration, i.e.,

$$
\boldsymbol{\Sigma}=\mathrm{E}\left[\boldsymbol{w}_{k-1}^{(r, V)}\left(\boldsymbol{w}_{k-1}^{(r, V)}\right)^{\mathrm{T}}\right]=\operatorname{diag}\left(\sigma_{x}^{2}, \sigma_{y}^{2}\right)
$$

where $\sigma_{x}$ and $\sigma_{y}$ are the process acceleration noise intensities.

Point current array is a constant matrix with a small noise. The whole state equation is

$$
\boldsymbol{x}_{k}=\boldsymbol{F} \boldsymbol{x}_{k-1}+\boldsymbol{w}_{k-1}
$$

where $\boldsymbol{F}=\left[\begin{array}{cc}\boldsymbol{\Phi}_{m} & \mathbf{0}_{5 \times N} \\ \mathbf{0}_{N \times 5} & \mathbf{I}_{N \times N}\end{array}\right]$; noise covariance matrix is $\boldsymbol{Q}=$ $\left[\begin{array}{c}\boldsymbol{\Gamma}_{m} \boldsymbol{\Sigma} \boldsymbol{\Gamma}_{m} \\ \alpha \mathbf{I}_{N \times N}\end{array}\right]$; and $\alpha$ is a small constant.
2.2. Moment Approximation Analysis. For nonlinear problems, the key that affects the Kalman-type filter algorithm is the approximation of the following observation characteristic moments.

$$
\begin{aligned}
& \widehat{\boldsymbol{h}}^{-}=\int_{\mathbb{R}^{m}} \boldsymbol{h}(\boldsymbol{x}) \mathrm{N}\left(\boldsymbol{x} ; \boldsymbol{x}_{0}, \boldsymbol{P}_{0}\right) \mathrm{d} \boldsymbol{x} \\
& \boldsymbol{P}_{h h}^{-} \\
& \quad=\int_{\mathbb{R}^{m}}\left(\boldsymbol{h}(\boldsymbol{x})-\widehat{\boldsymbol{h}}^{-}\right)\left(\boldsymbol{h}(\boldsymbol{x})-\widehat{\boldsymbol{h}}^{-}\right)^{\mathrm{T}} \mathrm{N}\left(\boldsymbol{x} ; \boldsymbol{x}_{0}, \boldsymbol{P}_{0}\right) \mathrm{d} \boldsymbol{x}
\end{aligned}
$$

where $\widehat{\boldsymbol{h}}^{-}$is the prior observation; $\boldsymbol{P}_{h h}^{-}$is the corresponding covariance. Formula (10) is the mean and covariance of the approximate Gauss distribution after the nonlinear transformation. Two typical moment approximation methods are considered:

(1) First Order (T1) Taylor Expansion:

$$
\begin{aligned}
\widetilde{\boldsymbol{h}}^{-} & =\boldsymbol{h}\left(\boldsymbol{x}_{0}\right) \\
\widetilde{\boldsymbol{P}}_{h h}^{-} & =\boldsymbol{H}_{0} \boldsymbol{P}_{0} \boldsymbol{H}_{0}^{\mathrm{T}}
\end{aligned}
$$

where $\boldsymbol{H}_{0}=\left.(\partial \boldsymbol{h} / \partial \boldsymbol{x})\right|_{\boldsymbol{x}=\boldsymbol{x}_{0}}$ is the Jacobian matrix.

(2) Unscented Transformation (UT) or Cubature Transformation (CT)

$$
\begin{aligned}
\widetilde{\boldsymbol{h}}^{-} & =\sum_{j=-n}^{n} \omega_{j} \mathscr{Y}_{j} \\
\widetilde{\boldsymbol{P}}_{h h}^{-} & =\sum_{j=-n}^{n} \omega_{j}\left(\mathscr{Y}_{j}-\widetilde{\boldsymbol{h}}^{-}\right)\left(\mathscr{Y}_{j}-\widetilde{\boldsymbol{h}}^{-}\right)^{\mathrm{T}}
\end{aligned}
$$

where $\mathscr{Y}_{ \pm j}=\boldsymbol{h}\left(\mathscr{X}_{ \pm j}\right), j=0,1, \cdots, n ; \mathscr{X}_{ \pm j}$ is the deterministic sampling point; and

$$
\begin{aligned}
X_{o} & =\boldsymbol{x}_{0}, \\
\mathscr{X}_{ \pm i} & =\boldsymbol{x}_{0} \pm \sqrt{(n+\lambda) \boldsymbol{P}_{0}} \\
\omega_{0} & =\frac{\lambda}{n+\lambda}, \\
\omega_{ \pm i} & =\frac{1}{2(n+\lambda)}
\end{aligned}
$$

where $i=1, \cdots, n$; for UT, $\lambda=3-n$; for CT $\lambda=0$.

We can get EKF, UKF, and CKF through these ways of approximation. For a general nonlinear model, suppose the Gaussian moment in the formula (10) could be solved accurately and the KLD between the joint Gaussian hypothesis distribution and the true distribution is [22]

$$
\begin{aligned}
& I(p, N)=\log (\mid \boldsymbol{I} \\
& \left.\quad+\boldsymbol{R}^{-1}\left(\boldsymbol{P}_{k \mid k-1}^{y y}-\left(\boldsymbol{P}_{k \mid k-1}^{x y}\right)^{\mathrm{T}}\left(\boldsymbol{P}_{k \mid k-1}\right)^{-1} \boldsymbol{P}_{k \mid k-1}^{x y}\right) \mid\right)
\end{aligned}
$$


When the priori estimation error $\boldsymbol{P}_{k \mid k-1}$ increases, the KLD between the approximate distribution and true distribution will increase, meaning that the Gaussian distribution is not a valid assumption any more, which will cause the filter to degrade or even divergence under large prior error conditions.

\section{Progressive Update Kalman Filters}

3.1. New Design for Measurement Update of Kalman Filters. The new algorithm focuses on redesigning measurement update. Time interval $\left[t_{k-1}, t_{k}\right]$ is divided into $N_{\mathrm{pu}}$ subintervals, and the interval of each subinterval is $\delta \lambda=\left(t_{k}-\right.$ $\left.t_{k-1}\right) / N_{\mathrm{pu}}$, which amount to obtaining $N_{\mathrm{pu}}$ observations sequentially in $\left[t_{k-1}, t_{k}\right]$. A measurement update process is performed in each of the subinterval. For time interval changes, observation noise covariance will also change.

It is easy to shown that the variance of the mean of $N_{\text {pu }}$ random independent zero-mean samples is equal to the variance of the individual samples divided by $N_{\mathrm{pu}}$, that is [23],

$$
\begin{aligned}
\mathrm{E}\left(\frac{1}{N_{\mathrm{pu}}} \sum_{i=1}^{N_{\mathrm{pu}}} \boldsymbol{r}_{i}\right)^{2} & =\frac{1}{N_{\mathrm{pu}}^{2}} \mathrm{E}\left[\boldsymbol{r}_{1}+\boldsymbol{r}_{2}+\ldots \boldsymbol{r}_{N_{\mathrm{pu}}}\right]^{2} \\
& =\frac{1}{N_{\mathrm{pu}}^{2}} \mathrm{E}\left[\boldsymbol{r}_{1}^{2}+\boldsymbol{r}_{2}^{2}+\ldots \boldsymbol{r}_{N_{\mathrm{pu}}}^{2}\right] \\
& =\frac{1}{N_{\mathrm{pu}}} \sigma_{r}^{2}
\end{aligned}
$$

where $\mathrm{E}\left[\boldsymbol{r}_{i}\right]=0, \mathrm{E}\left[\boldsymbol{r}_{i} \boldsymbol{r}_{j}\right]=\sigma_{r}^{2} \delta_{i j}$. Then the new observation noise covariance and the original noise covariance have the following relation:

$$
\boldsymbol{R}_{i}^{\text {(original) }}=\frac{1}{N_{\mathrm{pu}}} \boldsymbol{R}_{i}^{(\text {new })} \Longleftrightarrow \boldsymbol{R}_{i}^{(\text {new })}=N_{\mathrm{pu}} \boldsymbol{R}_{i}^{\text {(original) }}
$$

In the traditional Kalman filters, the EKF is the simplest in form. Therefore take PUEKF as a representative for PUKF. Measurement update for PUEKF in $[(i-1) \delta \lambda, i \delta \lambda]$ is as follows ( $\delta \lambda$ is omitted in the subscript):

$$
\begin{aligned}
\boldsymbol{S}_{i-1} & =\widehat{\boldsymbol{H}}_{i-1} \boldsymbol{P}_{i-1} \widehat{\boldsymbol{H}}_{i-1}^{\mathrm{T}}+N_{\mathrm{pu}} \boldsymbol{R}_{k} \\
\boldsymbol{K}_{i} & =\boldsymbol{P}_{i-1} \widehat{\boldsymbol{H}}_{i-1}^{\mathrm{T}} \boldsymbol{S}_{i-1}^{-1} \\
\widehat{\boldsymbol{x}}_{i} & =\widehat{\boldsymbol{x}}_{i-1}+\boldsymbol{K}_{i}\left(\boldsymbol{y}_{k}-\boldsymbol{h}\left(\widehat{\boldsymbol{x}}_{i-1}\right)\right) \\
\boldsymbol{P}_{i} & =\left(\boldsymbol{I}-\boldsymbol{K}_{i} \widehat{\boldsymbol{H}}_{i-1}\right) \boldsymbol{P}_{i-1}
\end{aligned}
$$

In $\left[t_{k-1}, t_{k}\right]$ the measurement update will be executed $N_{\mathrm{pu}}$ times. Then the PUEKF measurement update is as follows.

Steps 1. Initialization, $\widehat{\boldsymbol{x}}_{0}=\widehat{\boldsymbol{x}}_{k \mid k-1}, \boldsymbol{P}_{0}=\boldsymbol{P}_{k \mid k-1}$.

Steps 2. $i=1,2, \cdots, N_{\mathrm{pu}}$

Follow formula (17) to carry out measurement update.

Steps 3. $\widehat{\boldsymbol{x}}_{k \mid k}=\widehat{\boldsymbol{x}}_{N_{\mathrm{pu}}}, \boldsymbol{P}_{k \mid k}=\boldsymbol{P}_{N_{\mathrm{pu}}}$.
When $N_{\mathrm{pu}}=1$, the PUEKF algorithm degrades to the EKF algorithm.

From formula (17), we can see

$$
\boldsymbol{P}_{k \mid k-1}^{\left(N_{\mathrm{pu}}-1\right)}=\prod(\boldsymbol{I}-\boldsymbol{K} \boldsymbol{H}) \boldsymbol{P}_{k \mid k-1} \leq \boldsymbol{P}_{k \mid k-1}
$$

Through the analysis of formula (14) we know a large $\boldsymbol{P}_{k \mid k-1}$ is not conducive to filter stability, and the new algorithm reduces KLD by reducing $\boldsymbol{P}_{k \mid k-1}$, increasing the stability of the algorithm.

3.2. Linear Constraint for PUEKF. To match the point current array model to the actual situation, it is necessary to add linear constraint its state variables $\boldsymbol{x}$ :

$$
D x=d
$$

where $\boldsymbol{D}=\operatorname{diag}\left(\mathbf{0}_{1 \times 5}, \mathbf{I}_{1 \times N}\right), d \approx 0$.

The state is estimated as [24]

$$
\tilde{\boldsymbol{x}}=\operatorname{argmin} E\left(\|\boldsymbol{x}-\tilde{\boldsymbol{x}}\|^{2} \mid \boldsymbol{Y}\right), \quad \boldsymbol{D} \tilde{\boldsymbol{x}}=d
$$

Formula (20) ignores the time subscripts for random variables. The condition expectation is

$$
\begin{aligned}
\mathrm{E}\left(\|\boldsymbol{x}-\tilde{\boldsymbol{x}}\|^{2} \mid \boldsymbol{Y}\right)= & \int(\boldsymbol{x}-\tilde{\boldsymbol{x}})^{\mathrm{T}}(\boldsymbol{x}-\tilde{\boldsymbol{x}}) \operatorname{pdf}(\boldsymbol{x} \mid \boldsymbol{Y}) \mathrm{d} \boldsymbol{x} \\
= & \int \boldsymbol{x}^{\mathrm{T}} \boldsymbol{x} \operatorname{pdf}(\boldsymbol{x} \mid \boldsymbol{Y}) \mathrm{d} \boldsymbol{x} \\
& -2 \tilde{\boldsymbol{x}} \int \boldsymbol{x} \operatorname{pdf}(\boldsymbol{x} \mid \boldsymbol{Y}) \mathrm{d} \boldsymbol{x}+\tilde{\boldsymbol{x}}^{\mathrm{T}} \tilde{\boldsymbol{x}}
\end{aligned}
$$

The Lagrange expression is

$$
\begin{aligned}
\boldsymbol{L}= & \mathrm{E}\left(\|\boldsymbol{x}-\tilde{\boldsymbol{x}}\|^{2} \mid \boldsymbol{Y}\right)+2 \lambda_{\mathrm{L}}^{\mathrm{T}}(\boldsymbol{D} \tilde{\boldsymbol{x}}-d) \\
= & \int \boldsymbol{x}^{\mathrm{T}} \boldsymbol{x} \operatorname{pdf}(\boldsymbol{x} \mid \boldsymbol{Y}) \mathrm{d} \boldsymbol{x}-2 \widetilde{\boldsymbol{x}} \int \boldsymbol{x p d f}(\boldsymbol{x} \mid \boldsymbol{Y}) \mathrm{d} \boldsymbol{x} \\
& +\tilde{\boldsymbol{x}}^{\mathrm{T}} \tilde{\boldsymbol{x}}+2 \lambda_{\mathrm{L}}^{\mathrm{T}}(\boldsymbol{D} \tilde{\boldsymbol{x}}-d)
\end{aligned}
$$

To get the extreme value, the Lagrange partial derivative is set to be 0 , i.e.,

$$
\begin{aligned}
& \frac{\partial L}{\partial \widetilde{\boldsymbol{x}}}=-2 \widehat{\boldsymbol{x}}+2 \widetilde{\boldsymbol{x}}+2 \boldsymbol{D}^{\mathrm{T}} \lambda_{\mathrm{L}}=\mathbf{0} \\
& \frac{\partial L}{\partial \lambda}=\boldsymbol{D} \tilde{\boldsymbol{x}}-d=\mathbf{0}
\end{aligned}
$$

where $\widehat{\boldsymbol{x}}=\mathrm{E}(\boldsymbol{x} \mid \boldsymbol{Y})=\int \boldsymbol{x} \operatorname{pdf}(\boldsymbol{x} \mid \boldsymbol{Y}) \mathrm{d} \boldsymbol{x}$.

$\lambda_{\mathrm{L}}$ and $\tilde{\boldsymbol{x}}$ are available

$$
\begin{aligned}
\lambda_{\mathrm{L}} & =\left(\boldsymbol{D} \boldsymbol{D}^{\mathrm{T}}\right)^{-1}(\boldsymbol{D} \widehat{\boldsymbol{x}}-d) \\
\tilde{\boldsymbol{x}} & =\widehat{\boldsymbol{x}}-\boldsymbol{D}^{\mathrm{T}}\left(\boldsymbol{D} \boldsymbol{D}^{\mathrm{T}}\right)^{-1}(\boldsymbol{D} \widehat{\boldsymbol{x}}-d)
\end{aligned}
$$

Add Steps 4 to PU-EKF measurement update.

Steps 4. $\widehat{\boldsymbol{x}}_{k \mid k}=\widehat{\boldsymbol{x}}_{k \mid k}-\boldsymbol{D}^{\mathrm{T}}\left(\boldsymbol{D} \boldsymbol{D}^{\mathrm{T}}\right)^{-1}\left(\boldsymbol{D} \widehat{\boldsymbol{x}}_{k \mid k}-d\right)$

We get the complete measurement update steps for the linear constraint PUEKF (LPUEKF). 
TABLE 1: Simulation scene parameters.

\begin{tabular}{lc}
\hline Parameter/(unit) & Magnitude \\
\hline $\boldsymbol{r}_{0} /(\mathrm{m})$ & {$[-30,-30,12.5]$} \\
$\boldsymbol{V}_{0} /(\mathrm{m} / \mathrm{s})$ & {$[10,10]$} \\
$\boldsymbol{I}_{0} /(\mathrm{A})$ & {$[-5.05,5.05]$} \\
$l_{d} /(\mathrm{m})$ & 10 \\
$o_{1,2}$ & {$[0, \pm 5,0]$} \\
\hline
\end{tabular}

TABLE 2: Filter initial condition. The initial error is set to maximum to test the performance of the algorithm under large initial error conditions.

\begin{tabular}{lcc}
\hline Parameter/(unit) & Initial value & Initial mean square error \\
\hline$\widehat{\boldsymbol{r}}_{0} /(\mathrm{m})$ & {$\left[x_{0}, y_{0}, 12.51\right]$} & $\operatorname{diag}\left\{p_{0}^{2}, p_{0}^{2}, 0.01^{2}\right\}$ \\
$\widehat{\boldsymbol{V}}_{0} /(\mathrm{m} / \mathrm{s})$ & {$[10.2,10.2]$} & $\operatorname{diag}\left\{0.2^{2}, 0.2^{2}\right\}$ \\
$\widehat{\boldsymbol{I}}_{p} /(\mathrm{A})$ & $\mathbf{0}_{1 \times 2}$ & $\operatorname{diag}\left\{6^{2}, 6^{2}\right\}$ \\
\hline$* p_{0}=5,10,20,40\left(x_{0}, y_{0}\right)=(-35,-25),(-40,-20),(-50,-10),(-70,10)$.
\end{tabular}

\section{Simulation Results and Analysis}

4.1. A Demonstration Example. First, consider a demonstration example of tracking a point-current dipole consisting of two point currents. Consider the simulation scene shown in Table 1. Two electric field sensors are located at $o_{1,2}$. The initial value and initial mean square error of the filter are shown in Table 2. In addition, the process acceleration noise intensities $\sigma_{x}=0.1, \sigma_{y}=0.1$, and $\alpha=0.0001$; observation noise covariance $\boldsymbol{R}_{k}=\sigma^{2} \mathbf{I}_{2 \times 2}, \sigma=1 e-5$; progressive step of LPUEKF $\delta \lambda=0.05$; seawater depth $H=$ $60 \mathrm{~m}$; simulation time $T_{N}=41$. Choose the new algorithm LPUEKF and traditional Kalman filters (EKF, UKF, and CKF) as the tracking algorithms.

The root mean square error of $\boldsymbol{r}$ position component at $k$ moment $\left(R M S E_{k}^{r}\right)$ is chosen as performance metric, and it is formulated as

$$
\operatorname{RMSE}_{k}^{r}=\sqrt{\frac{1}{M C} \sum_{i=1}^{M C}\left\|\widehat{\boldsymbol{r}}_{k}^{(i)}-\boldsymbol{r}^{(i)}\right\|_{2}^{2}} ; \quad k=1, \cdots, T_{N}
$$

Figure 2 shows $R M S E_{k}^{r}$ under different initial error conditions.

As shown in Figure 2,

(1) LPUEKF obtains better results under different initial error conditions. In contrast, performance of traditional algorithms become poor as the initial error increases.

(2) when the target is far away from electric sensors, $R M S E_{k}^{r}$ increases with the decrease of SNR (signal to noise ratio), which is due to the fact that the dynamic estimation depends on the current observation information.

\subsection{A Simulation with Beasy Data}

4.2.1. Beasy Software Simulation of Ship UEP. Beasy software is used to evaluate the ship UEP during the ship design phase
TABLE 3: Simulation scene parameters.

\begin{tabular}{lc}
\hline Parameter/(unit) & Magnitude \\
\hline $\boldsymbol{r}_{0} /(\mathrm{m})$ & {$[-140,10,23.2]$} \\
$\boldsymbol{V}_{0} /(\mathrm{m} / \mathrm{s})$ & {$[15,0]$} \\
\hline
\end{tabular}

TABLE 4: Filter initial condition.

\begin{tabular}{lcc}
\hline Parameter/(unit) & Initial value & Initial mean square error \\
\hline$\widehat{\boldsymbol{r}}_{0} /(\mathrm{m})$ & {$[-420,90,23]$} & $\operatorname{diag}\left\{280^{2}, 80^{2}, 0.2^{2}\right\}$ \\
$\widehat{\boldsymbol{V}}_{0} /(\mathrm{m} / \mathrm{s})$ & {$[17,0.26]$} & $\operatorname{diag}\left\{3^{2}, 1^{2}\right\}$ \\
$\widehat{\boldsymbol{I}}_{p} /(\mathrm{A})$ & $\mathbf{0}_{1 \times 9}$ & $\mathbf{I}_{9 \times 9}$ \\
\hline
\end{tabular}

[25]. Consider a ship: the length $140 \mathrm{~m}$, the width $20 \mathrm{~m}$, and the draft depth $5 \mathrm{~m}$. The hull material is steel and the hull has a corrosion-resistant coating. The propeller material is coppernickel-aluminum alloy. Assuming that the hull coating is somewhere damaged and the propeller is exposed in the sea, so propeller and hull damage area is corroded area. Seawater conductivity $\sigma_{\text {sea }}=4 \mathrm{~s} / \mathrm{m}$, seabed conductivity $\sigma_{\text {sbed }}=$ $0.1 \mathrm{~s} / \mathrm{m}$, seawater depth $H=60 \mathrm{~m}$, and computed plane depth $h=23.2 \mathrm{~m}$ (relative to the sea level). Ship UEP data obtained from Beasy software is approximately consistent with the real ship UEP. Use these data as sensor observation information.

4.2.2. Simulation Conditions, Results, and Analysis. Consider the simulation scene shown in Figure 3. Three sensors array lay in line as $Y$-axis. $O$ is in the middle sensor. Point current array (the ship) is moving parallel to the $X$-axis. $Z$-axis is perpendicular to XOY and points to the sea surface. We also use $R M S E_{k}^{r}$ as the performance metric. At the same time, the length and width of the ship have great diversity, so the estimation error of $x$ and $y$ components should also be concerned. The root mean square errors of $x, y$ component at $k$ moment $\left(R M S E_{k}^{x}, R M S E_{k}^{y}\right)$ are the same as formula (25) in form.

The number of point current is 9 , and the distance $l_{d}=$ $16 \mathrm{~m}$. The center point of the point current array is taken as the reference point of tracking. The number of sensor is 3; distance between sensors is $20 \mathrm{~m} ; \boldsymbol{R}_{k}=\sigma^{2} \mathbf{I}_{3 \times 3}, \sigma=1 \times 10^{-4}$; progressive step of LPUEKF is 0.02 ; seawater depth $H=60 \mathrm{~m}$; simulation time $T_{N}=232 ; M C=100$. The other parameters are shown in Tables 3 and 4 .

The results are shown in Figure 4. UKF allows integral weights to be negative, and in the high dimensional tracking simulation, $\boldsymbol{P}$ is easy to be nonpositive, resulting in filtering anomalies. Therefore, UKF tracking result is not shown in the figure.

As shown in Figure 4, LPUEKF has better performance than the other algorithms. To further observe the LPUEKF tracking effect, the simulation results are displayed as shown in Figure 5. Figure 5(a) shows $R M S E_{k}^{\text {pos }}$ for $\boldsymbol{r}, x$, and $y$; Figure 5(b) shows one tracking trajectory of a simulation result.

In Figure 5(a), at moment $k=141, R M S E_{k}^{r}=30.09 \mathrm{~m}$, $R M S E_{k}^{x}=30.09 \mathrm{~m}$, and $R M S E_{k}^{y}=0.4668 \mathrm{~m}$. Compared with the ship scale in direction $x(140 \mathrm{~m}), y(20 \mathrm{~m})$, and the initial 

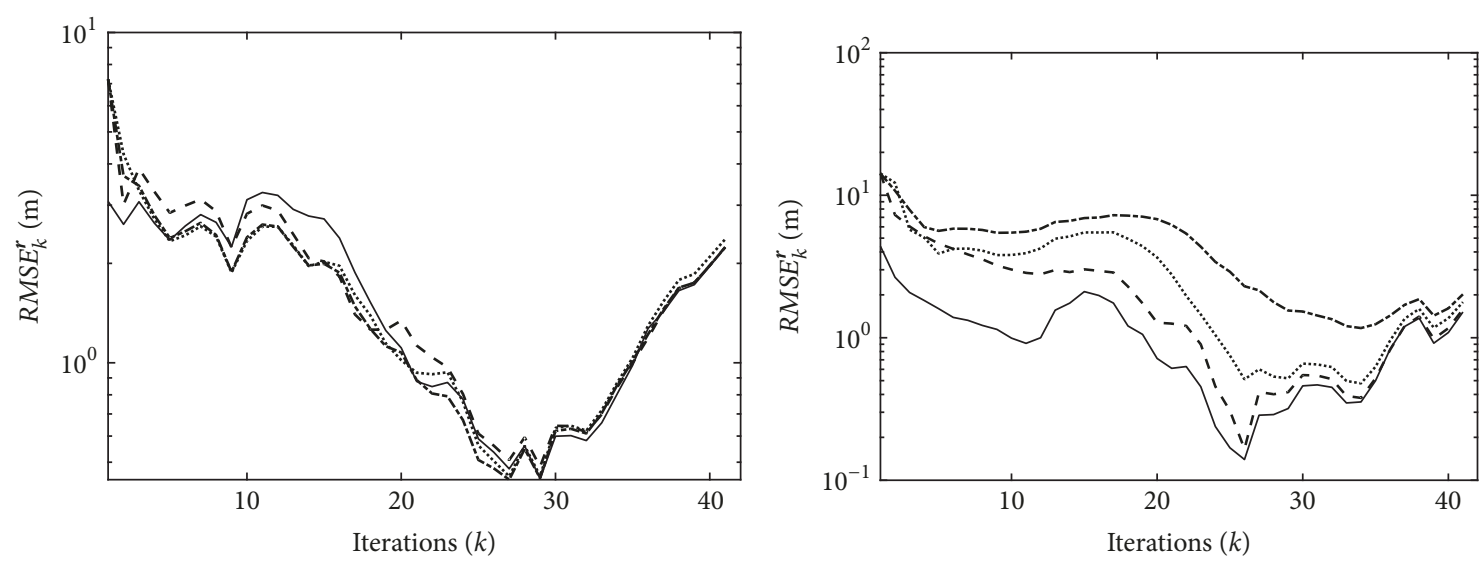

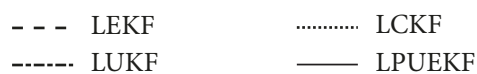

(a) $p_{0}=5$



(c) $p_{0}=20$

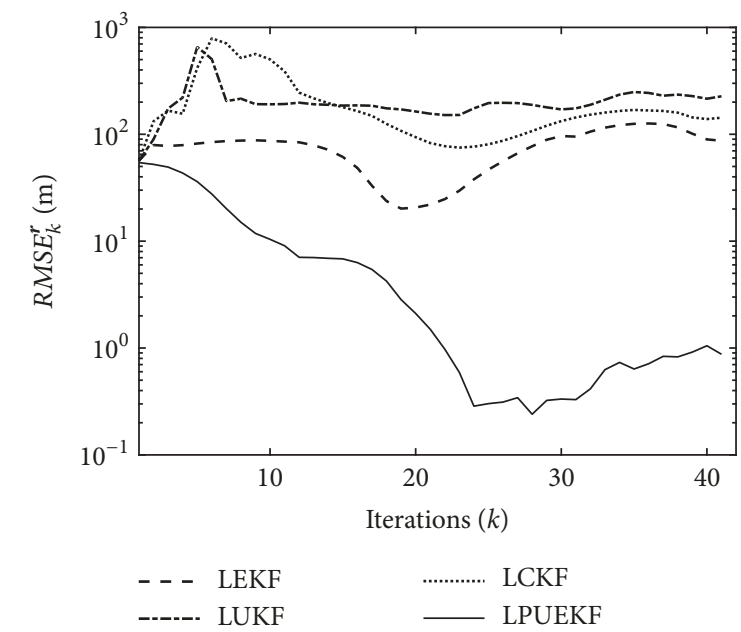

(d) $p_{0}=40$

FIGURE 2: $R M S E_{k}^{r}$ under different initial error conditions.

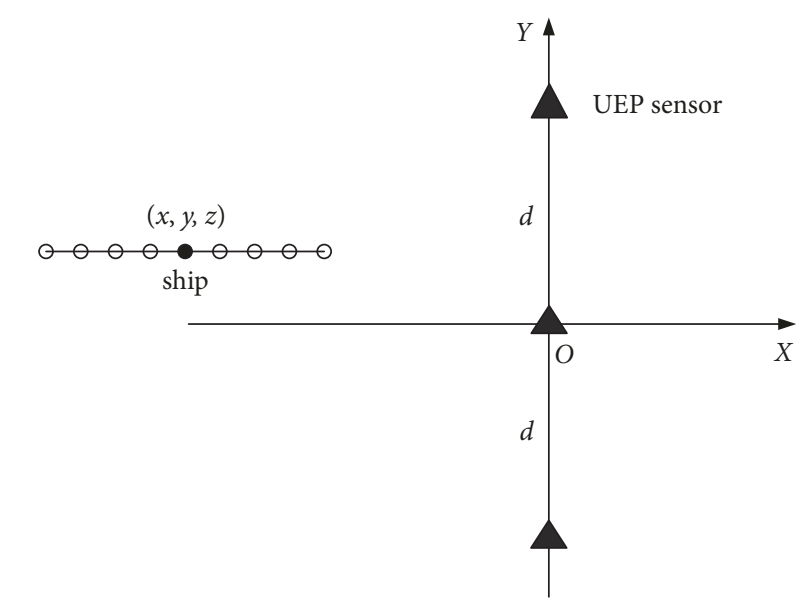

FIGURE 3: Projected diagram of ship passing through the electric field sensor array.

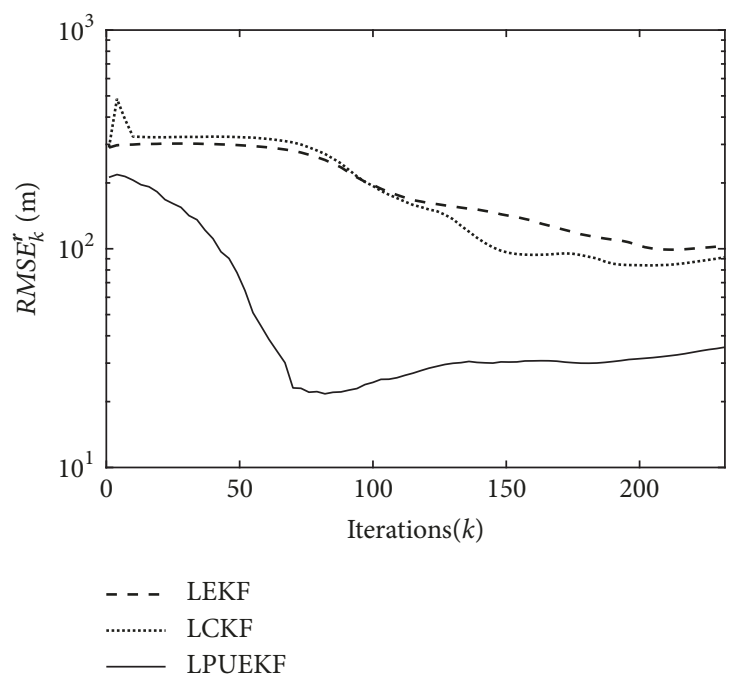

FIGURE 4: Tracking results of each algorithm. 


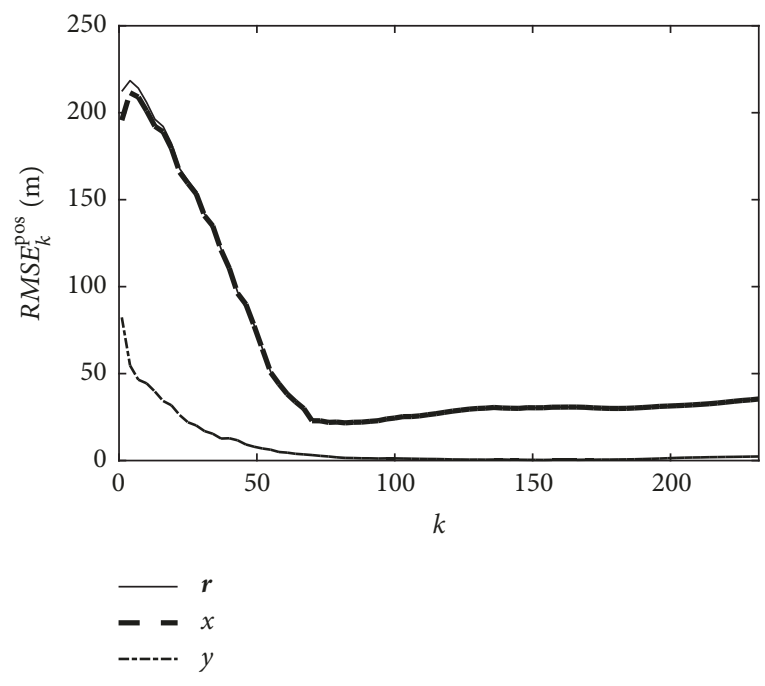

(a) $R M S E_{k}^{\mathrm{pos}}$ of $\boldsymbol{r}, x, y$

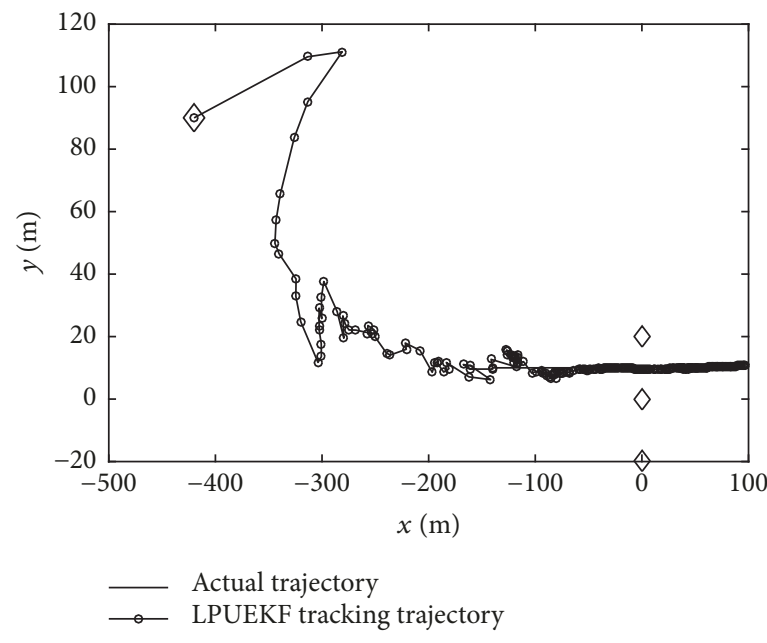

(b) One of LPUEKF tracking trajectory

FIGURE 5: LPUEKF tracking results.

error $(280 \mathrm{~m}, 60 \mathrm{~m})$, the tracking accuracy of the algorithm is enough to meet the demand. We can see from Figure 5(b) that LPUEKF can converge to the correct target position quickly after limited iterations.

The ship is described by 9 point currents whose initial value are $\mathbf{0}_{1 \times 9}$ and the center point of the point current array is taken as tracking reference point. As a result, local optimal solution inevitably exists in the filtering process. That is why the estimation of the $x$ direction cannot be as accurate as the estimate in the $y$ direction.

\section{Conclusions}

UEP is an important feature of a ship, which contains the location information of the ship. We study the possibility of using UEP to track the ship in this paper. In the current tracking algorithm, the filtering algorithm is the most suitable for real-time tracking. Among them, the Kalman filters are more suitable for underwater applications because of their easy programming and less computation. Aiming at the limitations of traditional Kalman filters in high-dimensional tracking problems, we propose progressive update Kalman filters based on progressive Bayesian. Taking PUEKF as a representative, the simulation shows that it has better convergence than traditional Kalman filters. The simulation with Beasy data demonstrates the good performance of the new algorithm and the possibility of using UEP to track a ship.

\section{Data Availability}

The data used to support the findings of this study are available from the corresponding author upon request.

\section{Conflicts of Interest}

The authors declare no conflicts of interest.

\section{Acknowledgments}

This study is sponsored by Science and Technology on NearSurface Detection Laboratory fund [TCGZ2017A007] and National Natural Science Foundation of China Youth Project [no. 51509252].

\section{References}

[1] C. Chen, Z. Jiang G, and L. F. Yao, "Characteristic analysis of corrosion-related static electromagnetic field produced by a submarine in shallow sea," Journal of Naval University of Engineering, vol. 26, no. 3, p. 1, 2014.

[2] A. Guibert, O. Chadebec, J.-L. Coulomb, and C. Rannou, "Ships hull corrosion diagnosis from close measurements of electric potential in the water," IEEE Transactions on Magnetics, vol. 45, no. 3, pp. 1828-1831, 2009.

[3] R. Adey and J. M. Baynham, "Predicting corrosion related signatures," Simulation of Electrochemical Processes II, vol. 54, pp. 213-223, 2007.

[4] B. Sun Q, B. Yan, and R. X. Jiang, "Application of ship static electric field to ship tracking and positioning," Journal of Unmanned Undersea Systems, vol. 26, no. 1, pp. 57-62, 2018 (Chinese).

[5] B. Ristic, S. Arulampalam, and N. Gordon, "Beyond the kalman filter-particle filters for tracking applications," IEEE Trans of Aerospace and Electronic Systems, vol. 19, no. 7, pp. 37-38, 2003.

[6] S. Julier, J. Uhlmann, and H. F. Durrant-Whyte, "A new method for the nonlinear transformation of means and covariances in filters and estimators," IEEE Transactions on Automatic Control, vol. 45, no. 3, pp. 477-482, 2000.

[7] I. Arasaratnam and S. Haykin, "Cubature kalman filters," IEEE Transactions on Automatic Control, vol. 54, no. 6, pp. 1254-1269, 2009.

[8] A. K. Mahalanabis and M. Farooq, "A second-order method for state estimation of non-linear dynamical systems," International Journal of Control, vol. 14, no. 4, pp. 631-639, 1971. 
[9] P. S. Maybeck, Stochastic Models, Estimation and Control, vol. 141, Academic Press, 1982.

[10] L. Perea, J. How, L. Breger, and P. Elosegui, "Nonlinearity in sensor fusion: Divergence issues in EKF, modified truncated SOF, and UKF," in Proceedings of the AIAA Guidance, Navigation, and Control Conference 2007, pp. 1863-1878, USA, August 2007.

[11] F. Gustafsson, "Particle filter theory and practice with positioning applications," IEEE Aerospace and Electronic Systems Magazine, vol. 25, no. 7, pp. 53-81, 2010.

[12] J. Steinbring and U. D. Hanebeck, "Progressive Gaussian filtering using explicit likelihoods," in Proceedings of the 17th International Conference on Information Fusion, FUSION 2014, Spain, July 2014.

[13] Y. Huang, Y. Zhang, N. Li, and L. Zhao, "Gaussian approximate filter with progressive measurement update," in Proceedings of the 54th IEEE Conference on Decision and Control, CDC 2015, pp. 4344-4349, Japan, December 2015.

[14] E. D. F. Melo, S. Maskell, and M. Fasiolo, "Stochastic particle flow for nonlinear high-dimensional filtering problems," Statistics, 2017.

[15] H. X. Zhang, S. H. Zhou, and M. S. Feng, "Progressive extended kalman filter," Acta Electronica Sinica, vol. 45, no. 1, pp. 213-219, 2017 (Chinese).

[16] N. G. Zamani, "Boundary element simulation of the cathodic protection system in a prototype ship," Applied Mathematics and Computation, vol. 26, no. 2, pp. 119-134, 1988.

[17] P. Doig and P. E. J. Flewitt, "A finite difference numerical analysis of galvanic corrosion for semi-infinite linear coplanar electrodes," Journal of The Electrochemical Society, vol. 126, no. 12, pp. 2057-2063, 1979.

[18] C. Chen, D.-G. Li, and S.-G. Gong, "The method of the extrapolation of the static electric field of ships," Journal of Harbin Engineering University, vol. 30, no. 6, pp. 719-722, 2009.

[19] R. Jiang, Y. Hu, and S. Gong, "Research on static electric field depth conversion method of ship based on point charge," Journal of Radio Science, vol. 29, no. 4, pp. 685-693, 2013 (Chinese).

[20] A. R. Adey, A. C. Brebbia, and S. M. Niku, "Application of boundary elements in corrosion engineering," in Electrical Engineering Applications, C. A. Brebbia, Ed., pp. 34-64, SpringerVerlag, Berlin, Germany, 1990.

[21] L. Mei-Qin and L. Jian, Advanced Theory and Application of Target Tracking, vol. 106, Chinese Science Publishing and Media Ltd, Beijing, China, 2015.

[22] M. R. Morelande and A. F. Garcia-fernandez, "Analysis of Kalman filter approximations for nonlinear measurements," IEEE Transactions on Signal Processing, vol. 61, no. 22, pp. 54775484, 2013.

[23] B. P. Gibbs, Advanced Kalman Filtering, Least-Squares And Modeling: A Practical Handbook, John Wiley, 2011.

[24] D. Simon, Optimal State Estimation, John Wiley and Sons, New York, NY, USA, 2006.

[25] R. Adey and J. Baynham, Predicting Corrosion Related Electrical and Magnetic Fields Using BEM, Underwater Defense Technology, Tracey Westwood, 2000. 


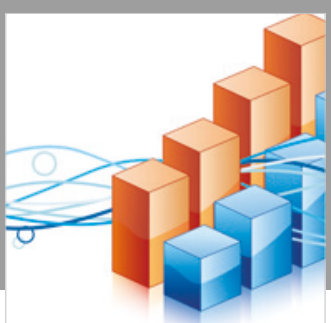

Advances in

Operations Research

\section{-n-m}
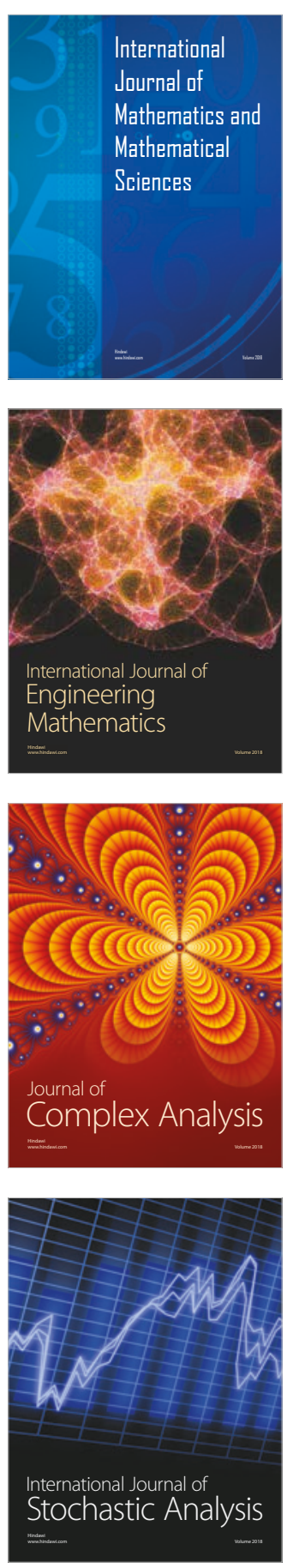
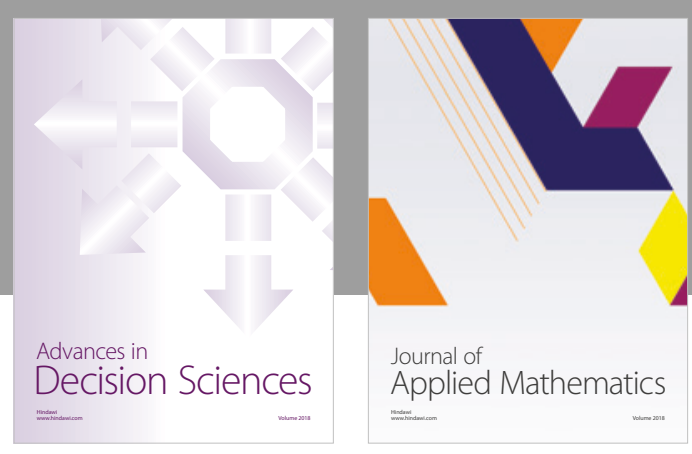

Journal of

Applied Mathematics
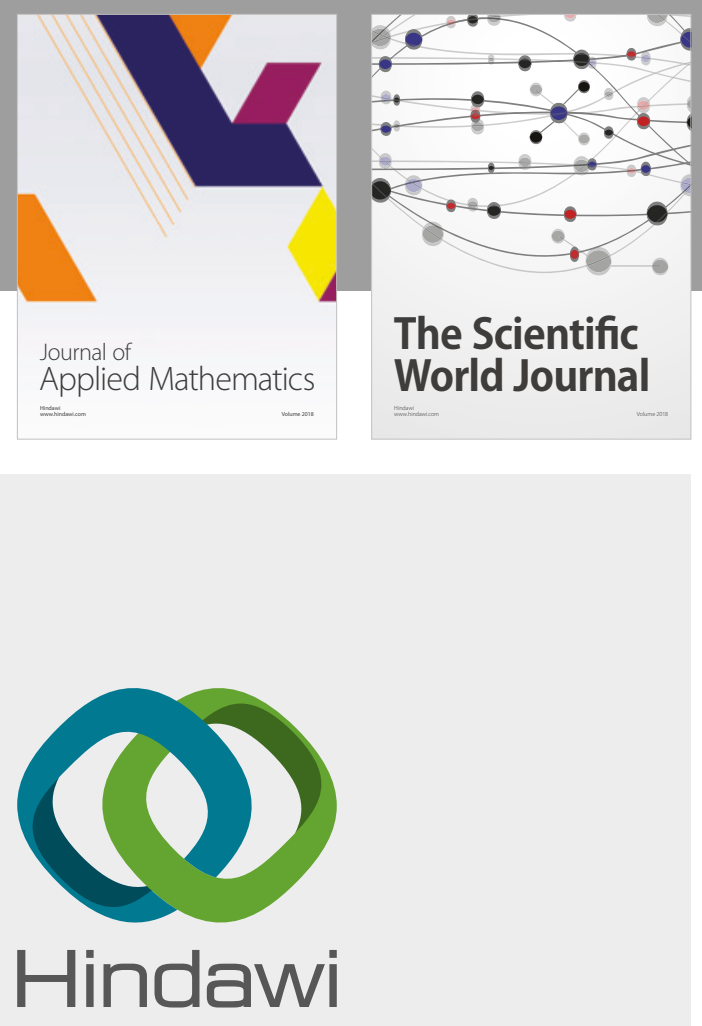

Submit your manuscripts at

www.hindawi.com

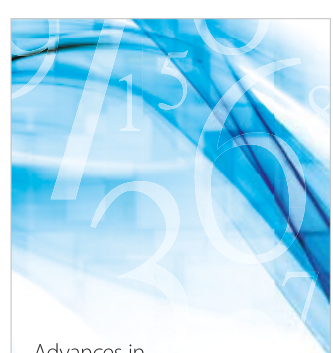

Advances in
Numerical Analysis


Mathematical Problems in Engineering



International Journal of

Differential Equations

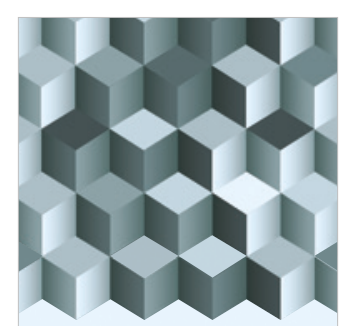

Journal of

Function Spaces

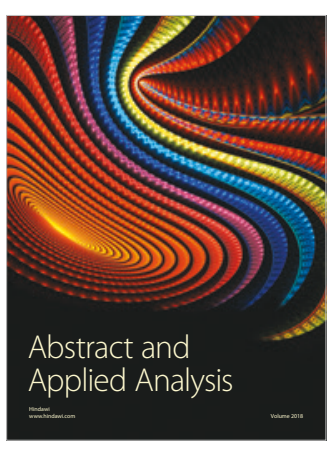

The Scientific

World Journal

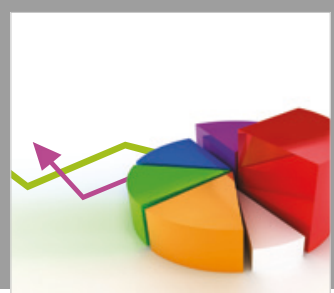

Journal of

Probability and Statistics
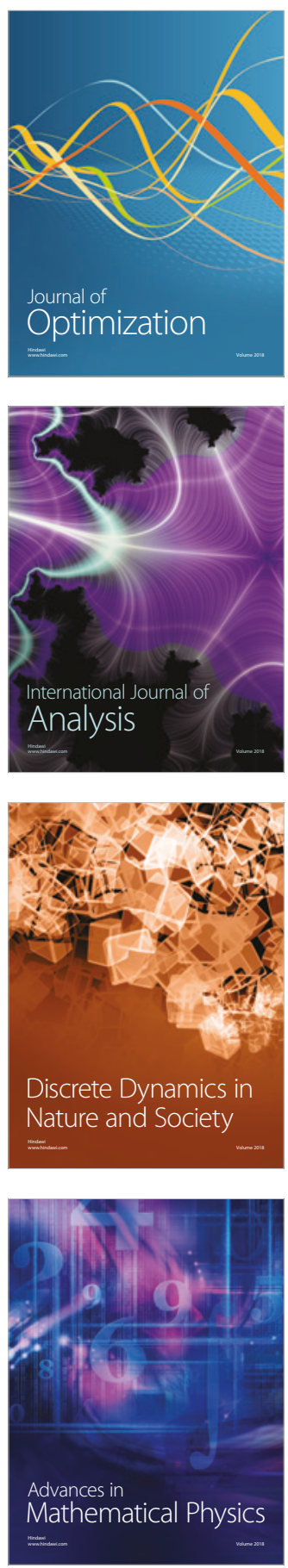